\title{
Impact of Intravitreal Ranibizumab Therapy on Vision Outcomes in Diabetic Macular Edema Patients: A Meta-Analysis
}

\author{
Manuel Falcão ${ }^{a, b}$

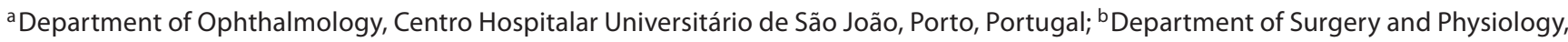 \\ Faculty of Medicine, University of Porto, Porto, Portugal
}

\section{Keywords}

Ranibizumab · Anti-VEGF therapy · Diabetic macular edema .

Clinical ophthalmology Clinical trials

\begin{abstract}
Purpose: Evaluation of the impact of the injection frequency of ranibizumab on best-corrected visual acuity (BCVA) outcomes in patients with diabetic macular edema (DME).

Methods: A meta-analysis of randomized controlled trials (RCTs) and real-world studies was performed to quantify the effect of ranibizumab treatment on BCVA and central foveal thickness (CFT) in DME as well as the relationship between the number of injections and the change in BCVA/CFT.
\end{abstract}

Results: All combined sources (29 RCTs and 11 real-life studies) showed a significant increase in BCVA from baseline following antivascular endothelial growth factor (VEGF) treatment (8.2, 9.4, and
10.3 ETDRS letters gained after 12, 24, and 36 months of ranibizumab treatment, respectively). The largest changes in BCVA were observed in RCTs and the smallest in real-life studies at 12 months. A significant relationship was found between the number of injections and change in BCVA at 12 months.

Conclusions: Inferior vision outcomes were observed in clinical practice compared with RCTs and might be partly attributable to administration of fewer anti-VEGF injections. Physicians should be aware that early and appropriate anti-VEGF treatment regimens are necessary to obtain the results reported in RCTs and help prevent irreversible vision loss in DME patients.

\section{Introduction}

Diabetic macular edema (DME) is a complex disease that is due to a multifactorial process involving the breakdown of the bloodretinal barrier and an abnormal accumulation of fluid in the retinal tissue of the macula [1]. Global statistics indicate that approximately $7.6 \%$ of people with diabetes may develop DME, but this figure can vary widely by geographical region [2]. DME is the most frequent cause of vision loss in patients with diabetic retinopathy and, untreated, can cause more than 10,000 new cases of blindness annually $[3,4]$. Furthermore, the majority of the estimated 26.7 million people worldwide living with DME are of working age $[2,5]$. Treatment of DME has been transformed in recent years by an improved understanding of the pathophysiological mechanisms leading to the condition, as well as by the development of effective treatments such as anti-vascular endothelial growth factor (VEGF) therapies, which can stabilize or improve vision in many patients [6-8]. Pivotal clinical trials evaluating intravitreal injections of anti-VEGF agents have reported clear benefits in visual and anatomic outcomes; as a result, this form of therapy has emerged as the preferred first-line treatment for DME, particularly in cases of foveal involvement and associated vision loss, thus displacing macular laser photocoagulation as standard of care [9]. Nevertheless, macular laser photocoagulation remains an important adjuvant treatment to improve macular thickness outcomes and reduce the number of anti- 
VEGF injections needed [7, 10]. Three anti-VEGF treatments are currently used in clinical practice to prevent/restore vision loss associated with DME: aflibercept $\left(\right.$ Eylea $^{\circledR}$ ), bevacizumab (Avas$\operatorname{tin}^{\circledR}$ ), and rani-bizumab (Lucentis ${ }^{\circledR}$ ) [11]. Ranibizumab and aflibercept are both approved for the treatment of visual impairment due to DME, while bevacizumab is not approved for ophthalmologic treatment but is used off-label by some physicians due to its lower costs [11].

Although anti-VEGF monotherapy with prompt or deferred fo$\mathrm{cal} /$ grid laser photocoagulation has been shown to be superior to laser therapy alone [10], it is not without limitations and side effects. DME persists in some patients, even after frequent antiVEGF intravitreal injections. In the RISE/RIDE phase III trials, DME persisted and central foveal thickness (CFT) remained $>250$ $\mu \mathrm{m}$ in approximately $23 \%$ of patients after 2 years of monthly ranibizumab injections [7].

Despite favorable effects of treatment, repeated injections may increase the risk of infectious endophthalmitis, intraocular inflammation, and possibly stroke or myocardial infarction [12]. Furthermore, the frequency of injections in a fixed monthly dosing regimen of anti-VEGF therapy may impose a significant burden on patients who either do not respond well or have persistent DME as well as on health care providers. To address these challenges and reduce the number of injections, studies have examined alternative dosing regimens, including "as needed" or "pro re nata" (PRN) regimens and "treat-and-extend" (TAE) strategies, in specific patient populations [13].

Mounting evidence from the literature suggests that the frequency of anti-VEGF injections correlates with efficacy [14]. In landmark clinical trials evaluating the efficacy and safety of ranibizumab (RESOLVE, RESTORE) [6, 10], bevacizumab (BOLT) [15], and aflibercept (VISTA and VIVID) [8] in patients with DME, the largest gains in best-corrected visual acuity (BVCA) were reported with frequent monitoring (monthly) and dosing (9-12 injections) during the first 12 months of treatment. However, such a fixed, monthly dosing regimen is associated with a huge socioeconomic burden for both the patient and the health care provider; hence, other regimens such as PRN and TAE regimens are more commonly adopted in real-world clinical practice [16]. Analysis of electronic medical records indicates that DME patients receiving anti-VEGF therapy in real-world clinical practice undergo less frequent monitoring, receive fewer intravitreal injections, and achieve inferior vision outcomes than patients in landmark clinical trials $[14,17,18]$. In clinical practice, Kiss et al. [14] reported that the mean number of anti-VEGF injections received by DME patients during their first year of treatment is only 2-4. In addition, an evaluation of everyday clinical practice data from the IRIS $^{\circledR}$ Registry in the USA showed that, among patients treated with anti-VEGF therapy within 28 days of their initial DME diagnosis, $50 \%$ receive only $1-3$ injections of anti-VEGF in their first year of treatment [19].

To the best of our knowledge, no systematic review has evaluated the impact of injection frequency on visual outcomes in patients with DME treated with ranibizumab injections in the real world and compared these with the outcomes reported in major clinical trials. We performed a systematic review and meta-analysis of randomized controlled trials (RCTs) and real-world studies reported to date, to quantify the effect of ranibizumab treatment on BCVA and CFT in DME after 1, 2, or 3 years of treatment. In addition, we report the relationship between the number of antiVEGF injections and the change in outcome, as well as between baseline outcomes and outcome improvement.

\section{Materials and Methods}

Identification and Selection of Eligible Studies

We searched PubMed and the Cochrane Database of Systematic Reviews for publications from June 2008 to 2018 to examine the literature on antiVEGF therapy in patients with DME. Our search was limited to human studies and full-text articles available in English. Of specific interest were studies of patients receiving ranibizumab. The systematic analysis focused on assessing the effect of therapy on BCVA and CFT after 1,2, or 3 years of treatment. Of additional interest was the relationship between the number of anti-VEGF injections and change in outcome, as well as the relationship between baseline outcome and outcome improvement.

\section{Exclusion Criteria}

Not all studies identified could be included in the data analysis. A number of reasons for exclusion were observed, the most frequent being the following:

1 Patients received more than one type of therapy, and results were not presented for ranibizumab separately.

2 Different patients underwent varying lengths of follow-up.

3 No information was available on the mean change in BCVA or CFT.

4 No information was available on the variation in the change in BCVA or $\mathrm{CFT}$; for example, the standard deviation, standard error, or $95 \%$ confidence interval (CI).

In addition, some studies could only be included in certain analyses based on the information provided; for example, a study providing data on the number of injections could be included in the meta-regression of this variable, but was excluded from the baseline BCVA meta-regression if no information on baseline BCVA was reported.

\section{Statistical Methods}

Individual studies were examined to obtain data on changes in BCVA and CFT after anti-VEGF therapy. Results were split according to the time of follow-up. The focus was on the results after 1,2, or 3 years of therapy. The first set of analyses used meta-analysis methods to examine the overall effects of treatment upon improvements in BCVA and CFT at each time point. In these analyses, the results from the different studies were pooled together to provide a single estimate of the differences in outcome between treatments. The meta-analysis weighted the studies according to the amount of information provided, specifically the standard error of the change in outcome. Typically, it would be expected that larger studies would have a smaller standard error. At each time point, the analyses were performed separately for RCTs and real-life studies, and then for all studies combined. The meta-analyses were performed using the DerSimonianLaird random-effect method, regardless of the amount of heterogeneity between studies. Notably, changes in BCVA of 10 letters have been shown to be clinically significant in a number of studies [20]. The amount of heterogeneity between studies was based on the significance of the betweenstudy heterogeneity, as well as on the size of the I2 value. Substantial heterogeneity was assumed if the I 2 value was $>50 \%$. A second set of analyses examined the relationship in the various studies between the number of injections and the change in outcome, as well as between the outcome at baseline and the change in outcome. These analyses were performed using meta-regression, again weighting the different studies according to the standard error of the change in outcome. Some studies provided data on different patient subgroups rather than the whole patient population. For these studies, each subgroup was regarded as a separate study in all analyses. 
Fig. 1. PRISMA-style flowchart of included and excluded ranibizumab studies. RWE, real-world evidence.

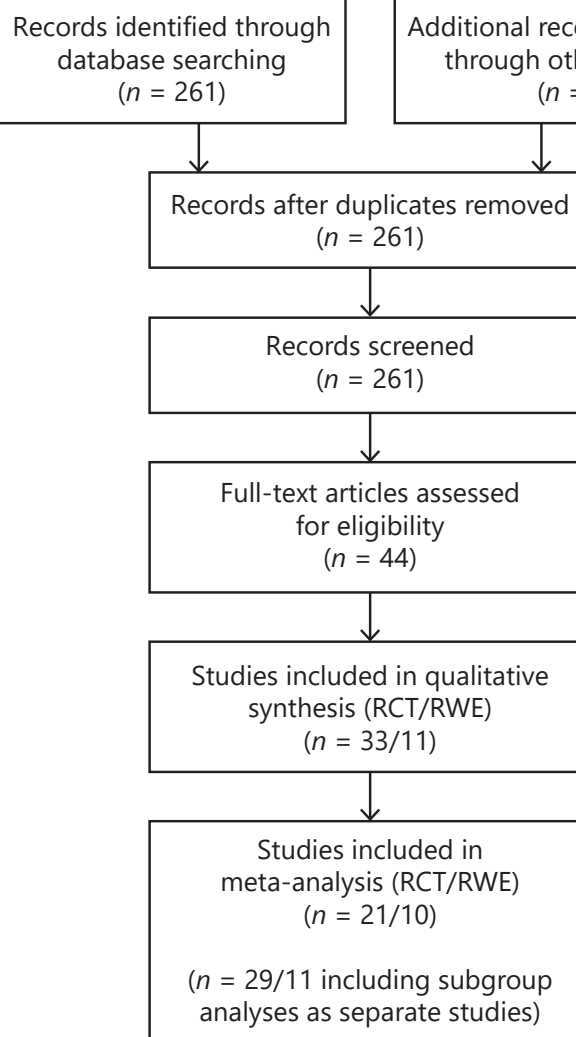

Records after duplicates removed $(n=261)$

Studies included in qualitative synthesis (RCT/RWE) $(n=33 / 11)$

Studies included in meta-analysis (RCT/RWE)

$(n=21 / 10)$

( $n=29 / 11$ including subgroup

analyses as separate studies)

Table 1. Pooled RCT and real-world study meta-analysis results for ranibizumab treatment showing mean change in BCVA at 12,24 , and 36 months

\begin{tabular}{|c|c|c|c|c|c|c|c|}
\hline \multirow[t]{2}{*}{ Time point } & \multirow{2}{*}{$\begin{array}{l}\text { Study } \\
\text { design }\end{array}$} & \multirow{2}{*}{$\begin{array}{l}\text { Studies }{ }^{a} \text {, } \\
n\end{array}$} & \multirow{2}{*}{$\begin{array}{l}\text { Injections }{ }^{b} \\
(\text { mean } \pm S D)\end{array}$} & \multicolumn{2}{|c|}{ Heterogeneity } & \multicolumn{2}{|l|}{ Treatment effects } \\
\hline & & & & $p$ value & $R^{2}$ & $\begin{array}{l}\text { mean change in ETDRS } \\
\text { letters }(95 \% \mathrm{Cl})\end{array}$ & $p$ value \\
\hline 12 months & $\begin{array}{l}\text { RCT } \\
\text { Real life } \\
\text { All }\end{array}$ & $\begin{array}{l}19 \\
11 \\
30\end{array}$ & $\begin{array}{l}8.5 \pm 2.3 \\
5.6 \pm 1.5 \\
7.4 \pm 2.5\end{array}$ & $\begin{array}{l}<0.001 \\
<0.001 \\
<0.001\end{array}$ & $\begin{array}{l}84 \% \\
96 \% \\
95 \%\end{array}$ & $\begin{array}{l}+9.1(8.0-10.3) \\
+6.3(4.5-8.1) \\
+8.2(6.9-9.4)\end{array}$ & $\begin{array}{l}<0.001 \\
<0.001 \\
<0.001\end{array}$ \\
\hline 24 months & $\begin{array}{l}\text { RCT } \\
\text { Real life } \\
\text { All }\end{array}$ & $\begin{array}{r}13 \\
1 \\
14\end{array}$ & $\begin{array}{l}10.0 \pm 5.1 \\
3.8 \\
9.5 \pm 5.2\end{array}$ & $\begin{array}{r}<0.001 \\
- \\
<0.001\end{array}$ & $\begin{array}{c}86 \% \\
- \\
86 \%\end{array}$ & $\begin{array}{l}+9.7(8.1-11.3) \\
+5.0(1.2-8.9) \\
+9.4(7.9-11.0)\end{array}$ & $\begin{array}{c}<0.001 \\
0.01 \\
<0.001\end{array}$ \\
\hline 36 months & $\begin{array}{l}\text { RCT } \\
\text { Real life } \\
\text { All }\end{array}$ & $\begin{array}{l}8 \\
0 \\
8\end{array}$ & $\begin{array}{c}17.1 \pm 12.9 \\
- \\
17.1 \pm 12.9\end{array}$ & $\begin{array}{c}0.003 \\
- \\
0.003\end{array}$ & $\begin{array}{c}79 \% \\
- \\
79 \%\end{array}$ & $\begin{array}{l}+10.3(8.4-12.2) \\
- \\
+10.3(8.4-12.2)\end{array}$ & $\begin{array}{r}<0.001 \\
- \\
<0.001\end{array}$ \\
\hline
\end{tabular}

Figures are not weighted by study size. Data are not available for all studies. BCVA, best-corrected visual acuity; Cl, confidence interval;

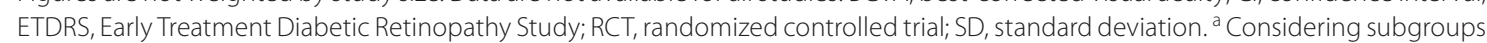
from the same study as separate studies. ${ }^{b}$ Raw summaries only.

\section{Results}

\section{Study Selection}

Electronic database search from June 2008 to 2018 was performed, and manual crosschecking of reference lists identified 267 articles for the initial review. After examination of all the files and ab- stracts by 1 reviewer, 44 articles satisfied the search criteria of anti-VEGF/ranibizu-mab therapy in patients with DME with available information on BCVA and/or CFT, and these went through a full-text review. After excluding studies in which patients received $>1$ type of therapy, and including subgroup analyses as separate studies, 40 studies (29 RCTs and 11 real-life studies) 
Fig. 2. Forest plot showing mean change in BCVA for ranibizumab studies at 12 months.

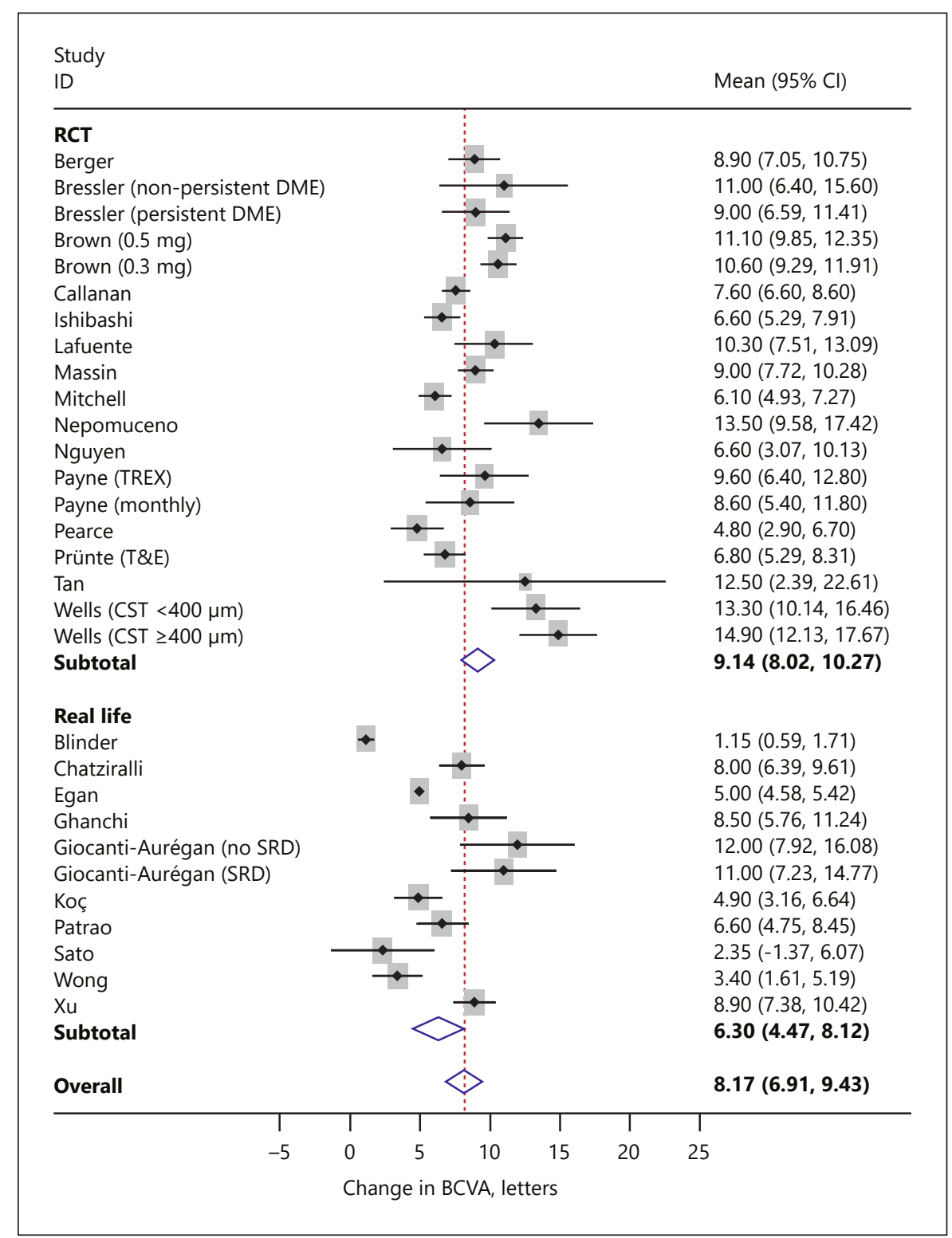

were included in this meta-analysis. Twenty-five of the 29 RCTs included were designed with a fixed monthly regimen of ranibizumab, whereas 7 of the 11 real-world studies analyzed involved a regimen of 3 consecutive monthly ranibizumab injections, followed by PRN treatment. The PRISMA flow diagram of study selection is shown in Figure 1. The characteristics of the 40 included studies are provided as online supplementary Tables (suppl. Table 1a-c; for all online suppl. material, see www.karger.com/ doi/10.1159/000505070, for studies with 12-, 24-, and 36-month data, respectively) $[6,10,12,13,20-48]$.

\section{BCVA Outcomes}

Meta-analysis was performed to examine the overall effect of ranibizumab on changes in BCVA at 12,24 , and 36 months. RCTs and real-world studies were analyzed separately, and results were then combined. The pooled results from the various studies are summarized in Table 1. Overall, there was signifi- cant heterogeneity between studies, with small $\mathrm{p}$ values $(\mathrm{p}<$ 0.001 ) and high $I 2$ values (79-95\%) suggesting a wide range of results across the different studies (Table 1). Given the varying characteristics, including patient disposition, number of injections, or treatment regimens (online suppl. Table $1 \mathrm{a}-\mathrm{c}$ ), in the different studies, variation in the results is not surprising. Results from the RCTs and real-world studies combined suggest that ranibizumab had a significant impact on BCVA at all time points evaluated (Table 1). At all time points, there was a significant increase in values compared with the pretreatment value. Considering all study types, the largest total increase from baseline was observed at 36 months, with a change in BVCA of 10 letters (Table 1).

At 12 months, an adequate number of both real-life studies and RCTs was available for analysis. At this time point, the largest changes in BCVA were observed in RCTs, with smaller effects seen in real-life studies (+9.1 vs. +6.3 ETDRS letters, respectively). 
Fig. 3. Meta-regression results for number of injections and change in BCVA for ran-ibizumab studies at 12 months.
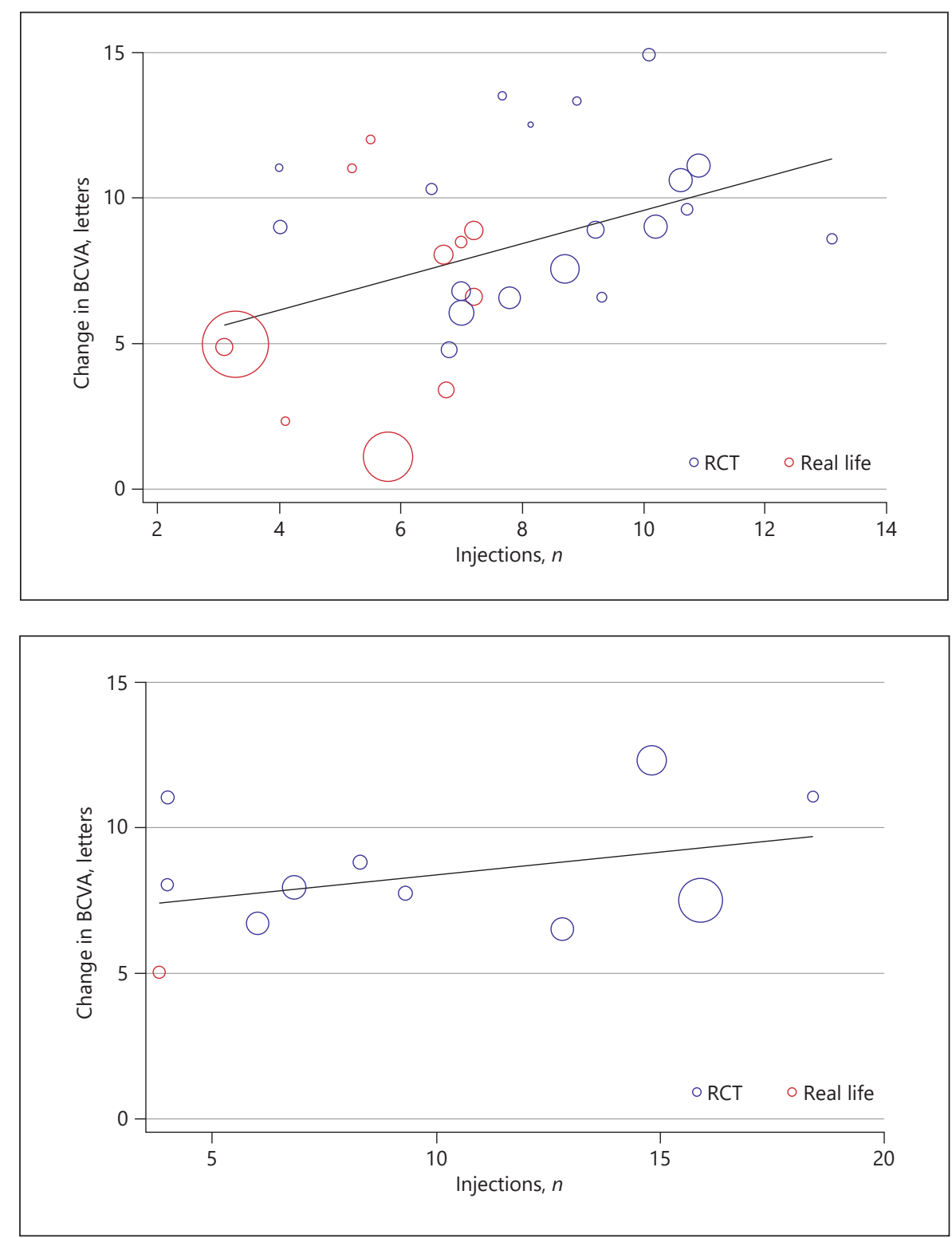

Fig. 4. Meta-regression results for number of injections and change in BCVA for ran-ibizumab studies at 24 months.
Insufficient real-life studies were available to compare BCVA with RCTs at the 24- and 36-month time points. The 12-month pooled results for each study type and all studies combined are shown in Figure 2.

\section{Injection Frequency and BCVA Outcomes}

Meta-regression analyses were used to examine how the number of ranibizumab injections and baseline BCVA were associated with the change in BCVA at 12 and 24 months. Insufficient data were available to evaluate this relationship at 36 months. The results for the number of injections suggest a significant relationship with change in BCVA at 12 months (fig. 3). There was a positive relationship, suggesting a greater number of injections was associated with a larger change in BCVA. An increase of 1 injection was associated with an increase of 0.6 letters. There was no significant relationship between baseline BCVA and the number of injections at 24 months (fig. 4).
There was no significant relationship between baseline BCVA and change in BCVA at either 12 or 24 months (data not shown). Insufficient data were available to properly evaluate this relationship at 36 months.

\section{CFT Outcomes}

Meta-analyses were performed to examine the overall effect of ranibizumab on changes in CFT at 12,24, and 36 months (please note, some of the studies included reported central subfield thickness, central macular thickness, central retinal thickness, central subfield macular thickness, or central retinal subfield thickness, all of which are included under CFT outcomes in the meta-analyses). These were performed for RCTs and real-world studies separately and combined. The pooled results from the various studies combined are summarized in Table 2.

The results from the various studies suggest that ran-ibizumab had a significant impact on CFT at all time points, showing a sig- 
Table 2. Meta-analysis results for ranibizumab studies showing mean changes in CFT from baseline to 12, 24, and 36 months

\begin{tabular}{|c|c|c|c|c|c|c|c|}
\hline \multirow[t]{2}{*}{ Time point } & \multirow{2}{*}{$\begin{array}{l}\text { Study } \\
\text { design }\end{array}$} & \multirow{2}{*}{$\begin{array}{l}\text { Studies } \\
n\end{array}$} & \multirow{2}{*}{$\begin{array}{l}\text { Injections }{ }^{\mathrm{b}} \\
(\text { mean } \pm \mathrm{SD})\end{array}$} & \multicolumn{2}{|c|}{ Heterogeneity } & \multicolumn{2}{|l|}{ Treatment effects } \\
\hline & & & & $p$ value & $R^{2}$ & $\begin{array}{l}\text { mean change in } \\
\text { ETDRS letters ( } 95 \% \mathrm{Cl})\end{array}$ & $p$ value \\
\hline 12 months & $\begin{array}{l}\text { RCT } \\
\text { Real life } \\
\text { All }\end{array}$ & $\begin{array}{r}15 \\
9 \\
24 \\
\end{array}$ & $\begin{array}{l}9.0 \pm 1.9 \\
5.6 \pm 1.5 \\
7.8 \pm 2.3 \\
\end{array}$ & $\begin{array}{l}<0.001 \\
<0.001 \\
<0.001\end{array}$ & $\begin{array}{l}94 \% \\
77 \% \\
92 \% \\
\end{array}$ & $\begin{array}{l}-159(-190 \text { to } 128) \\
-138(-158 \text { to } 119) \\
-152(-172 \text { to } 132)\end{array}$ & $\begin{array}{l}<0.001 \\
<0.001 \\
<0.001\end{array}$ \\
\hline 24 months & $\begin{array}{l}\text { RCT } \\
\text { Real life } \\
\text { All }\end{array}$ & $\begin{array}{l}6 \\
1 \\
7\end{array}$ & $\begin{array}{l}11.2 \pm 4.9 \\
3.8 \\
10.1 \pm 5.3\end{array}$ & $\begin{array}{l}<0.001 \\
- \\
<0.001\end{array}$ & $\begin{array}{l}81 \% \\
- \\
77 \%\end{array}$ & $\begin{array}{l}-136(-161 \text { to } 113) \\
-127(-161 \text { to } 93) \\
-135(-157 \text { to } 114)\end{array}$ & $\begin{array}{l}<0.001 \\
<0.001 \\
<0.001\end{array}$ \\
\hline 36 months & $\begin{array}{l}\text { RCT } \\
\text { Real life } \\
\text { All }\end{array}$ & $\begin{array}{l}5 \\
0 \\
5\end{array}$ & $\begin{array}{c}21.6 \pm 11.8 \\
- \\
24.7 \pm 10.1\end{array}$ & $\begin{array}{l}<0.001 \\
- \\
<0.001\end{array}$ & $\begin{array}{l}96 \% \\
- \\
96 \%\end{array}$ & $\begin{array}{l}-223(-278 \text { to } 169) \\
- \\
-223(-278 \text { to } 169)\end{array}$ & $\begin{array}{l}<0.001 \\
- \\
<0.001\end{array}$ \\
\hline
\end{tabular}

Figures are not weighted by study size. Data are not available for all studies. CFT, central foveal thickness; $\mathrm{Cl}$, confidence interval; RCT, randomized controlled trial; SD, standard deviation. ${ }^{a}$ Considering subgroups from same study as separate studies. ${ }^{b}$ Raw summaries only.

Table 3. Central foveal thickness (CFT) meta-regression results for combined randomized controlled trials (RCTs) and real-world ranibizumab studies

\begin{tabular}{|c|c|c|c|c|}
\hline Factor & Time point & Studies ${ }^{\mathrm{a}}, n$ & Coefficient (95\% Cl) & $p$ value \\
\hline Injections, $n$ & $\begin{array}{l}12 \text { months } \\
24 \text { months } \\
36 \text { months }\end{array}$ & $\begin{array}{r}25 \\
7 \\
6\end{array}$ & $\begin{array}{l}-9.6(-19.3 \text { to } 0.0) \\
-3.5(-8.7 \text { to } 1.8) \\
-5.3(-6.7 \text { to } 3.9)\end{array}$ & \\
\hline Baseline CFT & $\begin{array}{l}12 \text { months } \\
24 \text { months } \\
36 \text { months }\end{array}$ & $\begin{array}{r}25 \\
7 \\
2\end{array}$ & $\begin{array}{l}-0.65(-1.00 \text { to } 0.31) \\
0.33(-0.95 \text { to } 1.61) \\
b\end{array}$ & \\
\hline
\end{tabular}

Note: The regression coefficients provide the change in the outcome (CFT change) for a 1-unit increase in the explanatory factor; i.e., changes in outcome for a 1-injection increase, or a 1- $\mu \mathrm{m}$ increase in baseline CFT. Cl, confidence interval. ${ }^{\text {a }}$ Considering subgroups from the same study as separate studies. ${ }^{\mathrm{b}}$ No analysis performed due to an insufficient number of studies providing data.

nificant decrease compared with the baseline value. Considering all study types, the mean CFT decrease was $159 \mu \mathrm{m}$ at 12 months, $135 \mu \mathrm{m}$ at 24 months, and $223 \mu \mathrm{m}$ at 36 months. Notably, there was significant heterogeneity between studies, with low $\mathrm{p}$ values and high 12 values for all analyses.

At 12 months, an adequate number of both real-life studies and RCTs were available. At this time point, the changes in CFT were similar in RCTs compared with -real-life studies, albeit slightly greater in RCTs (Table 2; Fig. 5).

\section{Injection Frequency and CFT Outcomes}

A meta-regression approach was also used to examine the association between the number of injections and baseline CFT on the change in CFT at 12 and 24 months. Insufficient real-world data were available to properly evaluate this relationship at 36 months. The meta-regression results are summarized in Table 3.

The number of injections had an impact on the change in CFT at 12 and 36 months, although the association was only slightly significant at 12 months; the association was not deemed significant at 24 months. For the 12- and 36-month data, a greater number of injections was associated with a greater decrease in CFT. The largest effect was observed at 12 months, when an increase of 1 injection was associated with a $10-\mu \mathrm{m}$-larger decrease in CFT.

Baseline CFT was significantly associated with the change in CFT at 12 months. The negative regression coefficient suggests that a larger baseline CFT was associated with a greater reduction in CFT at 12 months. An increase of $1 \mu \mathrm{m}$ was associated with a $0.65-\mu \mathrm{m}$-larger decrease. This suggests that an increase of $100 \mu \mathrm{m}$ is associated with a $65-\mu \mathrm{m}$-larger decrease in CFT.

\section{Discussion and Conclusions}

In this meta-analysis, data from 29 RCTs and 11 real-world studies were analyzed to compare the effect of 1,2, or 3 years of antiVEGF treatment on functional and anatomical outcomes in patients with DME. Overall, our results support the use of intravitreal ranibizumab as an effective treatment option for the management of DME, demonstrating that good visual outcomes are achievable; for example, approximately 10 Early Treatment 


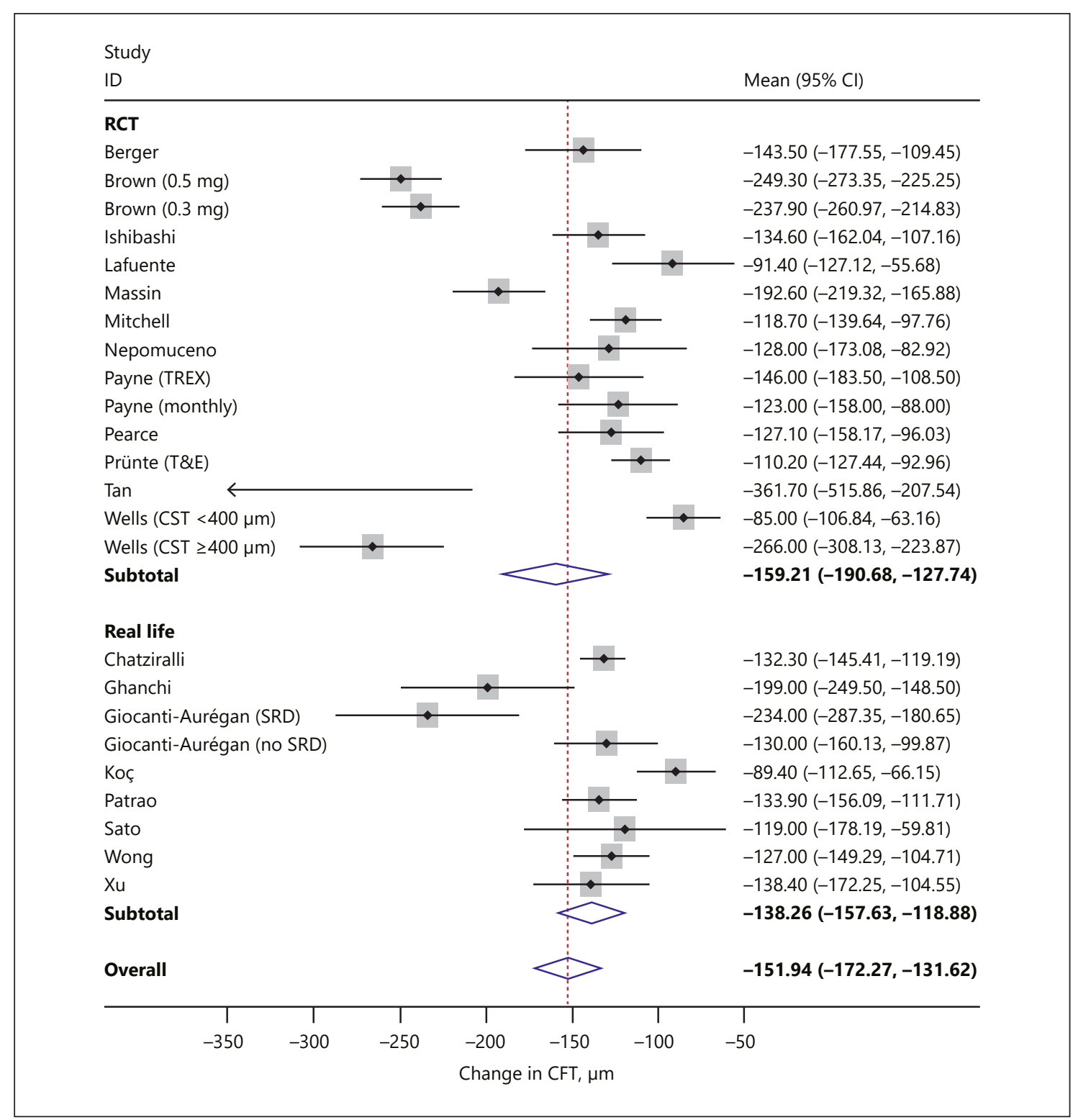

Fig. 5. Forest plot for ranibizumab studies at 12 months showing change in central foveal thickness (CFT) from baseline. CST, central subfield thickness; SRD, serous retinal detachment: T\&E/TREX, treat and extend.

Diabetic Retinopathy Study (ETDRS) letters may be gained over 3 years of treatment.

In key clinical trials, the mean improvement in BCVA with antiVEGF products has been reported to be in the range of 5-13 ETDRS letters after 1 year of treatment $[6,8,10,13,20,22,24,33,37$, 42, 48-52], 7-13 letters after 2 years of treatment [7, 12, 34, 43], and 8-14 letters after 3 years of treatment $[22,42,53]$. These improvements usually occur within 1 year and can be sustained for up to 5 years $[12,42,47]$. Our analysis of ranibizumab treatment showed a mean BVCA improvement within the range previously reported, with the largest change in BCVA occurring during the first 12 months of treatment and sustained to 3 years.

A substantial amount of evidence shows that eyes having a worse baseline BCVA achieve a greater mean visual improvement with
anti-VEGF therapy [54]. However, a correlation between baseline BCVA and change in BCVA at either 12 or 24 months was not shown in our analysis. This could have occurred due to the heterogeneity of patient characteristics in the studies analyzed, such as age and disease severity, comorbidities, methods for diagnosis and evaluation, follow-up, treatment doses and duration, and study design features, such as level of blinding.

Anti-VEGF injections tend to be given less frequently in clinical practice than in clinical trials and, as the number of such injections correlates with the degree of improvement in visual acuity, it is thought that the poorer outcomes observed in a real-life setting are at least partly attributable to the lower frequency of injections. Our results support this finding of a correlation between the frequency of anti-VEGF injections and efficacy [27]. Blinder 
et al. [27] reported that ranibizumab injections for DME are administered less frequently in clinical practice than recommended, resulting in less pronounced efficacy than observed in the clinical trials leading to registration of -ranibizumab. We found a statistically significant correlation between efficacy (i.e., visual acuity gain) and the number of ranibizumab injections in our metaanalysis. In general, patients receiving $>7$ intravitreal ranibizum$\mathrm{ab}$ injections had a greater BCVA gain than those who received < 7 (data not shown). In addition, the number of injections was generally greater in patients in RCTs, who had greater letter gains compared with patients in real-world studies. An analysis of 1 -year visual acuity outcomes from electronic medical records suggests that infrequent dosing might compromise the extent of vision improvement gained with anti-VEGF therapy during the first 12 months of treatment [18]. A comparison of DME trials shows that fixed dosing regimens [55] or strict PRN regimens with clear retreatment criteria [48] help to maximize the gains in BCVA, leading to optimal outcomes for patients. In contrast, the results from trials with less strict PRN treatment criteria $[10,13$, 24] show diminished BCVA gains and fewer injections in year 1 compared with trials with stricter regimens [48]. Similarly, in neovascular age-related macular degeneration, functional outcomes in real-world ranibizumab studies are less favorable with PRN regimens than fixed dosing regimens [56-58].

CFT, assessed by optical coherence tomography (OCT), provides a measure of retinal damage. Our current analysis shows a significant decrease in CFT in DME eyes successfully treated with anti-VEGF therapy. Pretreatment measurements of CFT have been reported to be a strong predictor of anatomical and functional outcomes in patients with DME treated with ranibizumab $[59,60]$. A higher baseline CFT was associated with a larger reduction in CFT after 1 year of anti-VEGF treatment, and vice versa, in our analysis.

Although evidence from the literature suggests a correlation between BCVA letter scores and CFT in DME patients treated with anti-VEGF agents, this correlation is only moderate during the first year of therapy $[59,61]$, implying that loss of visual acuity is likely multifactorial and might depend largely on the disruption of the retinal architecture or direct photoreceptor damage due to longstanding edema or other factors [62]. Some studies have shown that patients achieve excellent anatomic results with antiVEGF treatment but do not achieve corresponding functional improvement. Furthermore, disorganization of the inner retinal layers, determined by spectral-domain OCT, is associated with a worse baseline BCVA and less favorable outcomes [63]. Conversely, in some patients with persistent DME, BCVA may be maintained or improved following treatment. Our study highlights that more injections can result in better visual acuity despite reductions in macular thickness. Thus, the correlation between CFT and injection number in our study is probably clinically irrelevant. Indeed, in patients with DME, significant diurnal changes have been reported in retinal thickness measurements, which may be, in part, due to factors such as changes in blood pressure and retinal metabolism $[64,65]$. It is, therefore, possible to postulate that compared with maintenance of a continually thin retina by the administration of frequent anti-VEGF injections, fewer injections may lead to more pronounced retinal thickness fluctuations with worse visual outcomes.

Less frequent anti-VEGF injections during the first year of DME treatment in clinical practice result in less favorable visual outcomes compared with those seen over the same period in RCTs [18]. It is, therefore, increasingly important to reinforce, and educate on, the potential for undertreatment or delayed treatment to result in irreversible vision loss [66]. Clearly, physicians faced with suboptimal anti-VEGF effectiveness (i.e., a smaller than expected gain in vision, or persistent or recurrent macular edema) in everyday clinical practice could attempt to enhance effectiveness by increasing the number of anti-VEGF injections to more closely reflect the desired goal of monthly dosing. However, this may not be possible for a variety of reasons, including cost constraints, capacity issues in the clinic, and patient noncompliance due to the injection burden $[5,16]$. Overcoming these barriers is of paramount importance. Solutions may include having a larger number of injection clinics in which patients perform intravitreal treatments without an ophthalmologic observation in every visit. This would reduce the work burden without reducing the number of injections. Key trials in Europe have, therefore, evaluated alternative regimens with lower injection burdens while using a higher anti-VEGF dose (e.g., ranibizumab $0.5 \mathrm{mg}$ ) in a PRN approach as in the RESTORE trial [42], or a TAE regimen as used in the RETAIN trial [13]. A recent randomized study by Gillies et al. [51] supported a TAE regimen for achieving good visual outcomes with fewer visits for patients with neovascular age-related macular degeneration who have a highly variable need for retreatment. Furthermore, with the same mean number of 9.7 injections over 12 months, ranibizumab was shown to be noninferior to aflibercept for those patients [51].

Our study was limited by the following factors. The analyses showed considerable heterogeneity between study results, which could be regarded as a weakness of the study. However, the studies differed in various aspects, including the inclusion criteria and number of injections, so heterogeneity in outcomes might be expected. Results could also be affected by publication bias, with studies showing larger changes in outcome potentially being more likely to be published than those showing smaller effects. In summary, anti-VEGF injections are effective for many DME patients, but undertreatment in the real world may preclude patients from achieving the successful visual outcomes observed in RCTs. In our meta-analysis, an increase of 1 injection was associated with an increase of 0.6 letters and a decrease of 10 $\mu \mathrm{m}$ in CFT at 12 months. As more people are diagnosed with diabetes over the next 20 years, and the prevalence of DME is set to rise [2], it is increasingly important to improve effectiveness of DME treatments in the real-world setting; with ranibizumab, trying to mirror the number of treatments of the clinical trials is of paramount importance to obtain the expected results. 


\section{Acknowledgments}

Hayward Medical Communications and Klara Belzar (PhD) have drafted this manuscript as well as provided editorial support. Paul Bassett provided statistical support. Alimera Sciences Ltd funded the writing and project management and commented on the manuscript.

\section{Statement of Ethics}

This is a secondary analysis of published literature, and ethical approval is not required.

\section{Disclosure Statement}

The publication of this article was supported by Alimera Sciences Ltd. The author has been a consultant for Alimera and Bayer and participated in Advisory Boards for Novartis.

\section{Funding Sources}

Alimera Sciences Ltd funded the writing and project management and commented on the manuscript.

\section{Republication}

This article was first published in Ophthalmologica 2020;243:243-254.

\section{References}

References are available at www.karger.com/doi/10.1159/000511380. 